\title{
Modeling and Dynamics of HTS Motors for Aircraft Electric Propulsion
}

\section{Ranjan Vepa}

School of Engineering and Material Science, Queen Mary, University of London, London E14NS, UK; r.vepa@qmul.ac.uk; Tel.: +44-020-7882-5193

Received: 15 December 2017; Accepted: 12 February 2018; Published: 22 February 2018

\begin{abstract}
In this paper, the methodology of how a dynamic model of a conventional permanent magnet synchronous motor (PMSM) may be modified to model the dynamics of a high-temperature superconductor (HTS) machine is illustrated. Simulations of a typical PMSM operating under room temperature conditions and also at temperatures when the stator windings are superconducting are compared. Given a matching set of values for the stator resistance at superconducting temperature and flux-trapped rotor field, it is shown that the performance of the HTS PMSM is quite comparable to a PMSM under normal room temperature operating conditions, provided the parameters of the motor are appropriately related to each other. From these simulations, a number of strategies for operating the motor so as to get the propeller to deliver thrust with maximum propulsive efficiency are discussed. It is concluded that the motor-propeller system must be operated so as to deliver thrust at the maximum propulsive efficiency point. This, in turn, necessitates continuous tracking of the maximum propulsive efficiency point and consequently it is essential that the controller requires a maximum propulsive efficiency point tracking (MPEPT) outer loop.
\end{abstract}

Keywords: synchronous motor; superconductivity; high-temperature superconductor; dynamic modeling

\section{Introduction}

High-temperature superconductors were discovered in 1986 by two physicists, Johannes Georg Bednorz and Karl Müller, working at the International Business Machines (IBM) Labs. Bednorz and Müller [1] identified superconductivity in a sample of $\mathrm{LaBaCuO}$ material at the much higher temperature of $36 \mathrm{~K}$. Subsequently, high-temperature superconductivity was identified in structured materials containing planes of copper oxide, such as $\mathrm{YBa}_{2} \mathrm{Cu}_{3} \mathrm{O}_{7}$, by a group led by Chu [2]. These materials had a transition temperature beginning at $93 \mathrm{~K}$ and had zero resistance at $80 \mathrm{~K}$. The phenomenal growth in the research and development of devices and applications based on this effect have been documented by Ford and Saunders [3]. Yet, there are indeed a number of key issues that must be resolved before useful application products can be designed and mass produced. These issues include the problem of flux creep, weak links, and poor mechanical properties, which have also been identified by Goyal [4] and are being resolved.

An electrical motor can be termed as high temperature superconducting if its design contains elements that are high-temperature superconductors and these elements are working in the superconducting state at much higher temperatures than elements exhibiting the normal feature of electrical conduction. The motor usually contains a high-temperature superconducting element, such as an armature or a field winding or both. It is common practice to use a high current density and low loss high-temperature superconductor (HTS) wire for winding the armature or field coils or both. Although conventional superconducting materials with characteristically low resistance to electron flow were first discovered by the Dutch physicist Heike Kammerlingh Onnes in 1911, 
it was thought to have been completely explained in 1957 by the Bardeen, Cooper, and Schrieffer (BCS) theory $[5,6]$ based on quantum mechanics. The BCS theory completely failed to predict the existence of HTS, although it did provide a framework for characterizing HTS. A superconducting material is in the superconducting state when a point determined by the operating temperature, current density, and magnetic field characteristics lies below a surface defined by the material's critical characteristics. Critical surface characteristics are defined in terms of three variables: temperature, current density, and magnetic field intensity. Thus, superconductivity is generally observed below a critical temperature at a given critical (magnetic) field strength and a critical current density. A superconductor needs to be kept below a critical temperature $T_{\mathrm{c}}$ and the applied field must be less than the critical field $H_{\mathrm{c}}$. If an applied magnetic field is stronger than the critical field, it can penetrate the superconductor, causing a quenching of the superconducting state. Even though the temperature may be below the critical temperature, it will no longer be superconducting. Materials exhibiting this behavior are known as type I superconductors. However, HTS materials are known as type II, which exhibit two critical magnetic field states before losing the property of superconductivity. While the most well-known characteristic of superconductivity is zero resistance, the magnetic field inside a superconductor, which is proportional to the sum of the magnetization and the applied field, adds up to zero. The exclusion of magnetic fields within a superconductor is the defining characteristic of a superconductor and creates a phenomenon known as the Meissner effect. Thus, the disappearance of the electrical resistance and the Meissner effect when the magnetic field inside the superconductor is shielded by a lossless current flowing in a thin layer on the surface of the superconducting material (the perfect case of diamagnetism, which occurs when an external magnetic field penetrates only a finite, outer region of the material and does not influence the remaining inner domain of the material) are the fundamental properties of superconductors. Furthermore, in a cooled superconductor after the magnetization and the applied field add up to zero, if the applied field is removed, the magnetization remains and the internal field is now acting in a direction opposite to the originally applied field. Thus, the flux is essentially "trapped" within the superconductor. These superconductors are referred to as flux pinned or trapped superconductors and the trapped field could be as high as 15+ Tesla. The trapped field can be maintained, in principle, by a quantum process known as flux pumping whereby small amounts of magnetic flux are pumped in by the repeated application of magnetic transients, which require relatively low power.

Yttrium barium copper oxide (YBCO) is a family of crystalline chemical compounds known for exhibiting high-temperature superconductivity. This family of superconductors includes the first material that was discovered to become superconducting above the boiling point of liquid nitrogen $(77 \mathrm{~K})$ at about $93 \mathrm{~K}$. Typically, YBCO has a layered structure consisting of copper-oxygen planes with yttrium and barium atoms in the crystal structure as well. The resulting crystal structure is similar to a perovskite with a unit cell consisting of stacked cubes of $\mathrm{BaCuO}_{3}$ and $\mathrm{YCuO}_{3}$. $\mathrm{YBCO}$ conductors are manufactured as wire assemblies and as tapes that are now available from several industrial suppliers and are very promising conductors for the design of HTS electric motors and their applications. Many $\mathrm{YBCO}$ compounds have the general formula $\mathrm{YBa}_{2} \mathrm{Cu}_{3} \mathrm{O}_{7-x}$ (also known as Y123), although materials with other $\mathrm{Y}: \mathrm{Ba}: \mathrm{Cu}$ ratios exist, such as $\mathrm{YBa}_{2} \mathrm{Cu}_{4} \mathrm{O}_{y}(\mathrm{Y} 124)$ and $\mathrm{Y}_{2} \mathrm{Ba}_{4} \mathrm{Cu}_{7} \mathrm{O}_{y}$ (Y247). The bismuth strontium calcium copper oxide superconductor family (BSCCO tapes) - with the following compounds: $\mathrm{Bi}-2201$ or $\mathrm{Bi}_{2} \mathrm{Sr}_{2} \mathrm{CuO}_{6}, \mathrm{Bi}-2212$ or $\mathrm{Bi}_{2} \mathrm{Sr}_{2} \mathrm{CaCu}_{2} \mathrm{O}_{8}, \mathrm{Bi}-2223$ or $\mathrm{Bi}_{2} \mathrm{Sr}_{2} \mathrm{Ca}_{2} \mathrm{Cu}_{3} \mathrm{O}_{10}$-have also been used in the construction of HTS motors. The first HTS motors began appearing just before the end of the last millennium and in the following year [7-12]. Kalsi [13] has described in some detail the different types of superconducting machines and has provided models that could be used for simulation.

\section{Architectures of HTS Machines for Hybrid and All-Electric Propulsion}

Aircraft propulsion systems can benefit from electric HTS machines in two distinct ways. First, power can be generated by a conventional gas turbine and converted into electrical energy in an 
HTS generator, which in turn is used to power a controlled HTS motor driving a propeller. Due to the onboard availability of electric power, an electric motor may be used to drive the compressor in a high bypass ratio gas "turbine" engine, thus making the turbine redundant. Thus, such a hybrid propulsion system uses both HTS generators and motors. This turbo-generator motor drive has several advantages over a direct drive gas turbine propulsion system. The turbine main shaft speed is not limited by the fan and, therefore, can be operated at higher efficiencies. The use of a turbo-generator allows for greater redundancy, which in turn improves the overall reliability of the propulsion system. Moreover, a turbo-generator motor drive facilitates greater control of thrust and consequently makes propulsion-based flight control feasible, particularly when the gas turbine can be distributed along the lifting surfaces.

On the other hand, all-electric would use only HTS motors without the need for an onboard generator and would use only batteries or fuel cells to drive the propulsion HTS motors. A wide variety of HTS reluctance motors with diamagnetic rotors, HTS permanent magnets, and wound field synchronous motors and HTS brushless Direct Current (DC) motors are being developed for aircraft and other applications [14-20]. Thus, several superconducting field synchronous machines with room temperature or cryogenic high purity metal armatures are under development. In these applications, it is advantageous to reduce the number of moving parts in the HTS machines. For this reason, for most aircraft drive systems, rotary machines with HTS stator windings and permanent magnets or wound and trapped-field rotors are preferred. The stator is generally constructed by winding HTS tape, about 4-6 mm wide, flat on spools of the same width, over each of the poles, which are quite large. Such windings are referred to as pancake coils. Alternately, the windings are inserted into circular tracks on a disc and these types of windings are referred to as racetrack windings.

Most superconducting machines have employed liquid neon or cold gaseous helium for cooling HTS coils. Liquid nitrogen-cooled HTS motors have also been developed for ship propulsion by Okazaki, Sugimoto, and Takeda [21]. The coolant is generally transferred from an external refrigerator to the rotor through a rotating coupling. The field winding consists of several HTS coils that are conduction-cooled through the support structure or exposed to the tubes carrying the coolant to enhance the cooling rate. The torque tube is mainly responsible for transferring torque from the "cold" (cryogenically-cooled) environment to the "warm" shaft end. The pole pairs and the support structure are enclosed in the vacuum-sealed cryostat to minimize heat input due to radiation and to provide an insulated operating environment for the HTS field coils. A refrigeration system or cylinder, which uses the cold circulating gas in a closed loop, continuously maintains the HTS field winding at the desired low, cryogenic temperature.

One approach to keeping the field coils magnetized is to use flux-trapped superconductors to maintain the flux in the field coils. Such machines are modeled as high-field and low-resistance machines, but the torque constant is relatively high. They need minimal energy for super-cooling over an entire flight and continuous magnetization is provided by periodic flux pumping.

In this paper, the methodology of how a dynamic model of a conventional permanent magnet synchronous motor (PMSM) may be modified to model the dynamics of an HTS machine is illustrated. Simulations of a typical PMSM operating under room temperature conditions and at temperatures when the stator windings are superconducting are compared. Given a matching set of values for the stator resistance at superconducting temperature and flux-trapped rotor field, it is shown that the performance of the HTS PMSM is quite comparable to a PMSM under normal room temperature operating conditions, provided the parameters of the motors are appropriately related to each other. From these simulations, several strategies for operating the motor to get the propeller to deliver thrust with maximum propulsive efficiency are discussed. Thus, it is concluded that the motor-propeller system must be operated to deliver thrust at the maximum propulsive efficiency point. This, in turn, necessitates the continuous tracking of the maximum propulsive efficiency point and, consequently, it is essential that the controller requires a maximum propulsive efficiency point tracking (MPEPT) outer loop. 


\section{Dynamics of HTS Motors}

HTS motors- that is, motors with armature and field windings made of HTS wires or tapes that are encased within a cryostat so that the temperature of the stator and rotor are in the superconductivity domain-may be modeled just as conventional motors. However, the values of the resistances, torque, and back Electro-Motive Force (EMF) constants are substantially different from the corresponding values in a conventional motor. The models of DC, brushless DC (BLDC), and synchronous motors are briefly reviewed in the following subsections.

\subsection{Dynamics of an HTS DC Motor}

The mechanical shaft/armature dynamics of a DC motor are:

$$
J\left(d \omega_{m} / d t\right)=-B \omega_{m}+K_{T} i_{\text {drive }}-T_{L}, d \theta_{m} / d t=\omega_{m}
$$

where $\omega_{m}$ is the mechanical shaft speed, $\theta_{m}$ is the mechanical shaft angular position, $J$ is the shaft polar moment of inertia, $B$ is the viscous friction coefficient in the bearings, $K_{T}$ is the driving electrical torque constant, and $T_{L}$ is the load torque. Additionally, $i_{\text {drive }}=i_{a}$ is the rotor (armature) current, $L_{a}$ is the armature circuit equivalent inductance, and $R_{a}$ is the armature circuit equivalent resistance.

The armature equivalent circuit (current) dynamics are:

$$
L_{a}\left(d i_{a} / d t\right)=-R_{a} i_{a}+V_{i n}-K_{B} \omega_{m}
$$

where $V_{i n}$ is the input control voltage and $K_{B}$ is the back EMF constant. Ideally, $K_{T}=K_{B}$. In the case of an HTS motor, $R_{a}$ is considerably reduced while $K_{T}$ (and consequently $K_{B}$ ) is considerably increased.

\subsection{Dynamics of an HTS Brushless DC Motor}

A typical star connected three-phase BLDC motor is considered. The BLDC three-phase motor is driven by electronically switching on two of the three phases at a time, either in the forward or in the reverse direction while the remaining third phase is switched off.

The mechanical shaft/armature dynamics are:

$$
J\left(d \omega_{m} / d t\right)=-B \omega_{m}+T_{d r i v e}-T_{L}, d \theta_{m} / d t=\omega_{m}, \theta_{e}=P \times \theta_{m},
$$

where $\theta_{m}$ is the mechanical shaft angular position, $\theta_{e}$ is the electrical shaft position, $P$ is the number of pole pairs, $\omega_{m}$ is the mechanical shaft speed, $J$ is the shaft polar moment of inertia, $B$ is the viscous friction coefficient in the bearings, $T_{\text {drive }}$ is the driving electrical torque, and $T_{L}$ is the load torque. Additionally, $i_{a b}$ is the stator phase differential current, $v_{a b}$ is the stator phase differential voltage, $e_{a b}$ is the stator phase differential back EMF, $L_{S}$ is the stator phase circuit equivalent inductance, and $R_{S}$ is the stator phase circuit equivalent resistance. Thus, for a three-phase machine with three stator pole pairs,

$$
L_{s} \frac{d i_{a b}}{d t}=-R_{s} i_{a b}+v_{a b}-e_{a b}, L_{s} \frac{d i_{b c}}{d t}=-R_{s} i_{b c}+v_{b c}-e_{b c}, L_{s} \frac{d i_{c a}}{d t}=-R_{s} i_{c a}+v_{c a}-e_{c a}
$$

with,

$$
i_{a b}=i_{a}-i_{b}, i_{b c}=i_{b}-i_{c}, i_{c a}=i_{c}-i_{a}, e_{a b}=e_{a}-e_{b}, e_{b c}=e_{b}-e_{c}, e_{c a}=e_{c}-e_{a} .
$$

The back EMF in each phase is modeled as (Rambabu [22])

$$
e_{a}=\left(K_{B} / 2\right) \omega_{m} F_{1}\left(\theta_{e}\right), e_{b}=\left(K_{B} / 2\right) \omega_{m} F_{2}\left(\theta_{e}\right), e_{c}=\left(K_{B} / 2\right) \omega_{m} F_{3}\left(\theta_{e}\right) .
$$

The drive torque is

$$
T_{\text {drive }}=\left(K_{T} / 2\right)\left[\begin{array}{lll}
F_{1}\left(\theta_{e}\right) & F_{2}\left(\theta_{e}\right) & F_{3}\left(\theta_{e}\right)
\end{array}\right]\left[\begin{array}{lll}
i_{a} & i_{b} & i_{c}
\end{array}\right]^{T} \equiv\left(K_{T} / 2\right) \mathbf{F i}_{a b c}
$$


where each of the functions $F_{j}\left(\theta_{e}\right)$ is a trapezoidal function of $\theta_{e}$ given by

$$
\begin{aligned}
& F_{1}\left(\theta_{e}\right)=6 \theta_{e} / \pi \text { when } 0 \leq \theta_{e} \leq \pi / 6 \\
& F_{1}\left(\theta_{e}\right)=1 \text { when } \pi / 6 \leq \theta_{e} \leq 5 \pi / 6 \\
& F_{1}\left(\theta_{e}\right)=6-6 \theta_{e} / \pi \text { when } 5 \pi / 6 \leq \theta_{e} \leq 7 \pi / 6 \\
& F_{1}\left(\theta_{e}\right)=-1 \text { when } 7 \pi / 6 \leq \theta_{e} \leq 11 \pi / 6 \\
& F_{1}\left(\theta_{e}\right)=-12+6 \theta_{e} / \pi \text { when } 11 \pi / 6 \leq \theta_{e} \leq 12 \pi / 6 \\
& F_{2}\left(\theta_{e}\right)=-1 \text { when } 0 \leq \theta_{e} \leq 3 \pi / 6 \\
& F_{2}\left(\theta_{e}\right)=-4+6 \theta_{e} / \pi \text { when } 3 \pi / 6 \leq \theta_{e} \leq 5 \pi / 6 \\
& F_{2}\left(\theta_{e}\right)=1 \text { when } 5 \pi / 6 \leq \theta_{e} \leq 9 \pi / 6 \\
& F_{2}\left(\theta_{e}\right)=10-6 \theta_{e} / \pi \text { when } 9 \pi / 6 \leq \theta_{e} \leq 11 \pi / 6 \\
& F_{2}\left(\theta_{e}\right)=-1 \text { when } 11 \pi / 6 \leq \theta_{e} \leq 12 \pi / 6
\end{aligned}
$$

and

$$
\begin{aligned}
& F_{3}\left(\theta_{e}\right)=1 \text { when } 0 \leq \theta_{e} \leq \pi / 6 \\
& F_{3}\left(\theta_{e}\right)=2-6 \theta_{e} / \pi \text { when } \pi / 6 \leq \theta_{e} \leq 3 \pi / 6, \\
& F_{3}\left(\theta_{e}\right)=-1 \text { when } 3 \pi / 6 \leq \theta_{e} \leq 7 \pi / 6 \\
& F_{3}\left(\theta_{e}\right)=-8+6 \theta_{e} / \pi \text { when } 7 \pi / 6 \leq \theta_{e} \leq 9 \pi / 6 \\
& F_{3}\left(\theta_{e}\right)=1 \text { when } 9 \pi / 6 \leq \theta_{e} \leq 12 \pi / 6
\end{aligned}
$$

For a star connected motor, $i_{a}+i_{b}+i_{c}=i_{0}$. Using Equations (4) and (5), the complete three-phase BLDC motor state-space equations are obtained as follows:

$$
\begin{gathered}
\frac{d \theta_{m}}{d t}=\omega_{m}, J \frac{d \omega_{m}}{d t}=-B \omega_{m}+K_{T} i_{\text {drive }}-T_{L}, i_{\text {drive }}=\frac{1}{2} \mathbf{F i}_{a b c} \\
3 L_{s} \frac{d i_{a}}{d t}=-3 R_{s} i_{a}+2\left(v_{a b}-e_{a b}\right)+\left(v_{b c}-e_{b c}\right), 3 L_{s} \frac{d i_{b}}{d t}=-3 R_{s} i_{b}-\left(v_{a b}-e_{a b}\right)+\left(v_{b c}-e_{b c}\right),
\end{gathered}
$$

or

$$
\left[\begin{array}{cc}
L_{s} & 0 \\
0 & L_{s}
\end{array}\right] \frac{d}{d t}\left[\begin{array}{c}
i_{a} \\
i_{b}
\end{array}\right]=-\left[\begin{array}{cc}
R_{s} & 0 \\
0 & R_{s}
\end{array}\right]\left[\begin{array}{c}
i_{a} \\
i_{b}
\end{array}\right]+\frac{1}{3}\left[\begin{array}{cc}
2 & 1 \\
-1 & 1
\end{array}\right]\left[\begin{array}{c}
v_{a b}-e_{a b} \\
v_{b c}-e_{b c}
\end{array}\right] .
$$

In the case of an HTS motor, $R_{S}$ is considerably reduced while $K_{T}$ (and consequently $K_{B}$ ) is considerably increased.

\subsection{Dynamics of an HTS Synchronous Motor}

In a typical three-phase, two-pole permanent magnet synchronous motor (PMSM), the dynamic modeling begins with the choice of two reference frames. The three phases are labeled ' $a$ ' ' $b$ ' and ' $c$ ' respectively. The stator reference axis for the ' $a$ ' phase is generally chosen to the direction of maximum magneto-motive force or MMF, which is analogous to the EMF in electric circuits when a positive ' $a$ '-phase current is supplied at its maximum level. Reference axes for ' $b$ ' and ' $c$ ' stator phases are chosen $120^{\circ}$ and $240^{\circ}$ (electrical angle) ahead of the ' $a$ '-axis, respectively. Following the universal convention for choosing the rotor reference frame, the direction of permanent magnet flux is chosen as the $d$-axis, while the $q$-axis is $90^{\circ}$ ahead of the $d$-axis. Thus, one may also define a stator $d$-axis along the direction of the resultant stator field, while the stator $q$-axis is $90^{\circ}$ ahead of the stator $d$-axis. The angle of the rotor $d$-axis with respect to the stator $d$-axis is defined as $\theta_{r}$. The strength of the permanent magnetic field is defined by $\psi_{f}$.

From Vepa [23], in the stator $d$ - and $q$-axes,

$$
\left[\begin{array}{l}
u_{d s} \\
u_{q s}
\end{array}\right]=\left[\begin{array}{cc}
R_{d s} & 0 \\
0 & R_{q s}
\end{array}\right]\left[\begin{array}{c}
i_{d s} \\
i_{q s}
\end{array}\right]+\frac{d}{d t}\left[\begin{array}{c}
\lambda_{d} \\
\lambda_{q}
\end{array}\right],\left[\begin{array}{c}
\lambda_{d} \\
\lambda_{q}
\end{array}\right]=\left[\begin{array}{cc}
L_{d d} & L_{d q} \\
L_{d q} & L_{q q}
\end{array}\right]\left[\begin{array}{c}
i_{d s} \\
i_{q s}
\end{array}\right]-\psi_{f}\left[\begin{array}{c}
\cos \theta_{r} \\
\sin \theta_{r}
\end{array}\right]
$$


Differentiating the latter with time,

$$
\frac{d}{d t}\left[\begin{array}{l}
\lambda_{d} \\
\lambda_{q}
\end{array}\right]=\left[\begin{array}{ll}
L_{d d} & L_{d q} \\
L_{d q} & L_{q q}
\end{array}\right] \frac{d}{d t}\left[\begin{array}{c}
i_{d s} \\
i_{q s}
\end{array}\right]+\omega_{r} \psi_{f}\left[\begin{array}{c}
\sin \theta_{r} \\
-\cos \theta_{r}
\end{array}\right]
$$

Hence, it follows that

$$
\left[\begin{array}{l}
u_{d s} \\
u_{q s}
\end{array}\right]=\left[\begin{array}{cc}
R_{d s} & 0 \\
0 & R_{q s}
\end{array}\right]\left[\begin{array}{c}
i_{d s} \\
i_{q s}
\end{array}\right]+\left[\begin{array}{cc}
L_{d d} & L_{d q} \\
L_{d q} & L_{q q}
\end{array}\right] \frac{d}{d t}\left[\begin{array}{c}
i_{d s} \\
i_{q s}
\end{array}\right]+\psi_{f} \omega_{r}\left[\begin{array}{c}
\sin \theta_{r} \\
-\cos \theta_{r}
\end{array}\right] .
$$

In the rotor $\alpha$ - and $\beta$-axes, (stationary axes aligned with the stator $d$ - and $q$-axes) assuming $L_{d q}=0$, $R_{\alpha}=R_{d s}=R_{s}, R_{\beta}=R_{q s}=R_{s}, L_{\alpha}=L_{d d}, L_{\beta}=L_{q q}, u_{\alpha}=u_{d s}, u_{\beta}=u_{q s}, i_{\alpha}=i_{d s}, i_{\beta}=i_{q s}$, the rotor position $\theta_{m}$, speed $\omega_{m}$, and electrical frequency $\omega_{r}$ dynamic equations are as follows:

$$
d \theta_{r} / d t=\omega_{r}, d \theta_{m} / d t=\omega_{m}, \omega_{r}-P \omega_{m}=0, d \omega_{m} / d t=-(B / J) \omega_{m}+\left(T_{e m} / J\right)-\left(T_{L} / J\right) .
$$

The equation for the stator currents is as follows:

$$
\left[\begin{array}{cc}
L_{\alpha} & 0 \\
0 & L_{\beta}
\end{array}\right] \frac{d}{d t}\left[\begin{array}{l}
i_{\alpha} \\
i_{\beta}
\end{array}\right]+\left[\begin{array}{cc}
R_{\alpha} & 0 \\
0 & R_{\beta}
\end{array}\right]\left[\begin{array}{l}
i_{\alpha} \\
i_{\beta}
\end{array}\right]=\left[\begin{array}{ll}
1 & 0 \\
0 & 1
\end{array}\right]\left[\begin{array}{l}
u_{\alpha} \\
u_{\beta}
\end{array}\right]+\psi_{f} \omega_{r}\left[\begin{array}{c}
\sin \theta_{r} \\
-\cos \theta_{r}
\end{array}\right]
$$

To relate the stator currents to the three phase currents, Clarke's transformation from the stator three phase to the rotor $\alpha$ - and $\beta$-axes may be applied and this gives the following:

$$
\left[\begin{array}{l}
i_{\alpha} \\
i_{\beta} \\
i_{0}
\end{array}\right]=\frac{1}{\sqrt{6}}\left[\begin{array}{ccc}
2 & -1 & -1 \\
0 & \sqrt{3} & -\sqrt{3} \\
\sqrt{2} & \sqrt{2} & \sqrt{2}
\end{array}\right]\left[\begin{array}{c}
i_{a} \\
i_{b} \\
i_{c}
\end{array}\right]=\frac{1}{\sqrt{3}}\left[\begin{array}{ccc}
2 & 0 & -1 \\
0 & \sqrt{3} & 0 \\
1 & 0 & 2
\end{array}\right] \times \frac{1}{\sqrt{2}}\left[\begin{array}{ccc}
\sqrt{2} & 0 & 0 \\
0 & 1 & -1 \\
0 & 1 & 1
\end{array}\right]\left[\begin{array}{l}
i_{a} \\
i_{b} \\
i_{c}
\end{array}\right] .
$$

Similar equations hold for the phase voltages and voltages along the $\alpha$-and $\beta$-axes. Applying Parks transformation to transform from the rotor $\alpha$-and $\beta$-axes to the rotor $d$ - and $q$-axes gives the following:

$$
\left[\begin{array}{l}
i_{d} \\
i_{q} \\
i_{0}
\end{array}\right]=\left[\begin{array}{ccc}
\cos \theta_{r} & \sin \theta_{r} & 0 \\
-\sin \theta_{r} & \cos \theta_{r} & 0 \\
0 & 0 & 1
\end{array}\right]\left[\begin{array}{c}
i_{\alpha} \\
i_{\beta} \\
i_{0}
\end{array}\right],\left[\begin{array}{c}
i_{d} \\
i_{q}
\end{array}\right]=\left[\begin{array}{cc}
\cos \theta_{r} & \sin \theta_{r} \\
-\sin \theta_{r} & \cos \theta_{r}
\end{array}\right]\left[\begin{array}{c}
i_{\alpha} \\
i_{\beta}
\end{array}\right] \equiv \mathbf{T}\left[\begin{array}{c}
i_{\alpha} \\
i_{\beta}
\end{array}\right]
$$

Similar equations hold for the voltages along the $\alpha$ - and $\beta$-axes and the voltages along the $d$ - and $q$-axes. Using Equations (19) and (20) in the rotor's $d$ - and $q$-axes with $L_{\alpha}=L_{\beta}$ and $R_{\alpha}=R_{\beta}$, and with

$$
\left[\begin{array}{l}
u_{q} \\
u_{d}
\end{array}\right] \equiv \mathbf{T}\left[\begin{array}{l}
u_{\alpha} \\
u_{\beta}
\end{array}\right], \mathbf{T}\left[\begin{array}{c}
\sin \theta_{r} \\
-\cos \theta_{r}
\end{array}\right]=\left[\begin{array}{c}
0 \\
-1
\end{array}\right],
$$

one has for the rotor and current dynamics

$$
\begin{gathered}
\frac{d \theta_{m}}{d t}=\omega_{m}, \frac{d \omega_{m}}{d t}=-\frac{B}{J} \omega_{m}+\frac{T_{e m}}{J}-\frac{T_{L}}{J}=-\frac{B}{J} \omega_{m}+\frac{3 P\left(\psi_{f}+\left(L_{d}-L_{q}\right) i_{d}\right) i_{q}}{2 J}-\frac{T_{L}}{J} \\
{\left[\begin{array}{cc}
L_{q} & 0 \\
0 & L_{d}
\end{array}\right] \frac{d}{d t}\left[\begin{array}{c}
i_{q} \\
i_{d}
\end{array}\right]+\left[\begin{array}{cc}
R_{q} & \omega_{r} L_{d} \\
-\omega_{r} L_{q} & R_{d}
\end{array}\right]\left[\begin{array}{c}
i_{q} \\
i_{d}
\end{array}\right]=\left[\begin{array}{c}
u_{q} \\
u_{d}
\end{array}\right]+\left[\begin{array}{c}
-\omega_{r} \\
0
\end{array}\right] \psi_{f}=\left[\begin{array}{c}
u_{q}-\omega_{r} \psi_{f} \\
u_{d}
\end{array}\right] .}
\end{gathered}
$$

In general, for any of the DC, BLDC, or synchronous motors,

$$
\frac{d \theta_{m}}{d t}=\omega_{m}, J \frac{d \omega_{m}}{d t}=-B \omega_{m}+K_{T} i_{d r i v e}-T_{L}, i_{\text {drive }}=f(\mathbf{i}), \mathbf{L} \frac{d}{d t} \mathbf{i}=-\mathbf{R i}+\mathbf{G}\left(\mathbf{v}-\mathbf{e}_{\text {back }}\right) .
$$


In a typical flux-trapped HTS motor, $\mathbf{R}$ is reduced by a significant factor due to the property of superconductivity, while $K_{T}$ and $\mathbf{e}_{\text {back }}$ are both significantly increased due to the flux trapping. If only $\mathbf{R}$ is reduced by a significant factor, the motor would not be very stable in operation. However, because of the introduction of flux trapping, both $K_{T}$ and $\mathbf{e}_{\text {back }}$ are significantly and proportionately increased and, consequently, the stability of the motor is not degraded.

\section{Propeller Load Torque}

The blade element momentum (BEM) theory described in Vepa [23] is briefly summarized. It combines two methods of modeling the operation of a propeller where the first method is based on the balance of momentum within a rotating annular stream tube passing through a propeller disc, while the second method, which is known as the blade element theory, employs the airfoil lift and drag forces along various spanwise sections of the blade. Both methods are utilized to obtain the induced flow velocities across the propeller while only the second method is applied to obtain the blade thrust and blade torque.

In the blade element theory applied to a propeller blade, the blade is modeled by many elements along its length. On each element, following Vepa [23], it is assumed that:

(i) Aerodynamic interference between any of the blade elements is absent;

(ii) Only the two-dimensional section lift and drag forces are utilized to evaluate the forces on the blade elements.

To apply the BEM theory, following Vepa [23], the blade is divided into $N$ elements along its length. A slightly different flow is experienced at each blade element as they have different translational speeds. Furthermore, the chord lengths and inflow angles are different at each element. Following the division of the blade into a large number (usually about twenty or more) of elements, the blade element theory involves calculating the flow about each of the elements. By numerically integrating the forces and moments along the blade span, performance characteristics of the entire blade, such as torque, thrust and power coefficients, are obtained.

From Vepa [21], the load torque $T_{L}$ due to an $N$ bladed propeller may be written in terms of the blade element momentum (BEM) theory as follows:

$$
T_{L}=\frac{1}{2} \pi \rho \omega_{m}^{2} R^{5} \int_{r_{h}}^{1} \frac{\sigma^{\prime} \lambda_{f}^{2}(1+a)^{2} C_{D}}{\sin \phi \tan \phi}\left(1-\frac{C_{L}}{C_{D}} \tan \phi\right) \bar{r}^{2} d \bar{r} \equiv \frac{1}{8 \pi} \rho \omega_{m}^{2}(2 R)^{5} C_{T} .
$$

The propeller blade is divided into a finite number of blade sections, $N_{s}$, with uniform section properties and each with a length $\Delta r_{j}$ and writing the integral as a summation across the length of the blades from the hub to the tip,

$$
T_{L}=\frac{1}{2} \pi R^{3} \rho V_{w}^{2} \sum_{j=1}^{N_{s}} \frac{\sigma_{j}^{\prime}\left(1+a_{j}\right)^{2}}{\sin \phi_{j} \tan \phi_{j}}\left(C_{D j}-C_{L j} \tan \phi_{j}\right) \bar{r}_{j}^{2} \Delta \bar{r}_{j},
$$

where at a point $r$ along the radius of the propeller blade with blade chord $c$, blade length $R$ and a total of $N$ blades,

$$
\Delta \bar{r}_{j}=\Delta r_{j} / R, \bar{r}_{j}=r_{j} / R, \lambda_{f}=V_{w} / \omega_{m} R, \sigma_{j}^{\prime}=N c\left(\bar{r}_{j}\right) / \pi R \bar{r}_{j} .
$$

The axial and radial induction factors satisfy the following:

$$
\begin{gathered}
\frac{a_{j}}{\left(1+a_{j}\right)}=\frac{\sigma_{j}^{\prime}}{8 Q_{t i p, j}\left(\sin ^{2} \phi_{j}\right)}\left(C_{L j} \cos \phi_{j}+C_{D j} \sin \phi_{j}\right), \\
\frac{a_{j}^{\prime}}{\left(1+a_{j}\right)}=\frac{\sigma_{j}^{\prime}}{8 Q_{t i p, j} \bar{j}_{j}\left(\sin ^{2} \phi_{j}\right)} \lambda_{f}\left(-C_{L j} \sin \phi_{j}+C_{D j} \cos \phi_{j}\right), \phi=\tan ^{-1}\left(\lambda_{f}\left(1+a_{j}\right) / \bar{r}_{j}\left(1-a_{j}^{\prime}\right)\right),
\end{gathered}
$$


where $Q_{t i p, j}$ is the Prandtl's tip flow correction factor at a section defined as

$$
Q_{t i p, j}=(2 / \pi) \cos ^{-1}\left(f_{\text {loss }, j}\right), f_{\text {loss }, j}=N\left(\bar{r}_{j}^{-1}-1\right) /\left(2 \sin \phi_{j}\right) .
$$

The airfoil section drag coefficient is known to be composed of the profile and induced drag components and is assumed to be given by an expression representing a drag polar of the form:

$$
C_{D_{j}}=C_{D_{0}}-C_{D_{1}} C_{L_{j}}+C_{D_{2}} C_{L_{j}}^{2} \text { when }\left|\alpha_{j}\right|<\alpha_{s}
$$

and

$$
C_{D_{j}}=C_{D_{0 s}}-C_{D_{1 s}} \cos 2\left(\alpha_{j}-\alpha_{z}\right) \text { when }\left|\alpha_{j}\right| \geq \alpha_{s}
$$

where $\alpha_{s}$ is the value of $\alpha$ when stall is initiated and is determined by setting $d C_{L} / d \alpha=0$. Furthermore, $\alpha_{j}=\theta_{j}-\phi_{j}$, and $\theta_{j}$ is the local blade pitch angle (including the blade twist). Differentiating $\phi$ with respect to time gives the following:

$$
\dot{\phi}=\frac{\tan \phi}{\sec ^{2} \phi}\left(\frac{\dot{V}_{w}}{V_{w}}-\frac{\dot{\omega}_{m}}{\omega_{m}}+\frac{\dot{a}_{j}}{1+a_{j}}+\frac{\dot{a}_{j}^{\prime}}{1-a_{j}^{\prime}}\right) .
$$

Assuming that $a_{j}$ and $a_{j}^{\prime}$ are slowly varying functions and that

$$
\frac{\dot{a}_{j}}{1+a_{j}}+\frac{\dot{a}_{j}^{\prime}}{1-a_{j}^{\prime}} \cong 0,
$$

one may approximate

$$
\dot{\phi}=\frac{\tan \phi}{\sec ^{2} \phi}\left(\frac{\dot{V}_{w}}{V_{w}}-\frac{\dot{\omega}_{m}}{\omega_{m}}\right) .
$$

\section{Dynamic Model of Motor-Propeller System}

According to the Snel model, the lift coefficient is expressed as follows (see also Han, et al. [24]):

$$
C_{L}=C_{L}(\text { steady })+\Delta C_{L_{1 j}}+\Delta C_{L_{2 j}}
$$

In Equation (36), $\Delta C_{L_{1 j}}, \Delta C_{L_{2 j}}$ satisfy

$$
\begin{gathered}
\frac{d \Delta C_{L_{1 j}}}{d t}+C_{10 j} \Delta C_{L_{1 j}}=F_{1 j}(\psi), \\
\frac{d^{2} \Delta C_{L_{2 j}}}{d t^{2}}+C_{21 j}\left(\Delta C_{L_{2 j}}\right) \frac{d \Delta C_{L_{2 j}}}{d t}+C_{20 j}\left(\Delta C_{L_{2 j}}\right) \Delta C_{L_{2 j}}=F_{2 j}(\psi),
\end{gathered}
$$

where at a point $r_{j}$ along the radius of the blade with blade chord $c$ and blade length $R$,

$$
\tau_{j}=b_{j} / V_{r}=c\left(\bar{r}_{j}\right) / 2 R \omega_{m} \bar{r}_{j}, \bar{r}_{j}=r_{j} / R \text { and } \psi=\omega_{m} t .
$$

The coefficients of the model are defined as follows:

$$
\begin{gathered}
C_{10 j}=\frac{1+0.5 \Delta C_{L_{0 j}}}{8 \tau_{j}\left(1+60 \omega_{m} \tau_{j} d \alpha_{j} / d \psi\right)} \text { if } 2 \pi \sin \left(\alpha-\alpha_{z}\right) d \alpha / d \psi \leq 0, \\
C_{10}=\frac{1+0.5 \Delta C_{L_{0 j}}}{8 \tau\left(1+80 \omega_{m} \tau d \alpha / d \psi\right)} \text { if } 2 \pi \sin \left(\alpha-\alpha_{z}\right) d \alpha / d \psi \leq 0,
\end{gathered}
$$




$$
\begin{gathered}
\Delta C_{L_{0 j}}=2 \pi \sin \left(\alpha_{j}-\alpha_{z}\right)-C_{L}(\text { steady }), \\
C_{21 j}\left(\Delta C_{L_{2 j} j}\right)=12\left\{2\left(\Delta C_{L_{2 j}}\right)^{2}-0.01\left(\Delta C_{L_{0 j}}-0.5\right)\right\} \text { if } d \alpha_{j} / d \psi>0, \\
C_{21 j}\left(\Delta C_{L_{2 j}}\right)=0.4 \text { if } d \alpha_{j} / d \psi \leq 0, \\
C_{20 j}\left(\Delta C_{L_{2 j}}\right)=\left(0.04 / \tau^{2}\right)\left\{1+3\left(\Delta C_{L_{2 j} j}\right)^{2}\right\}\left(1+3\left(\omega_{m} d \alpha_{j} / d \psi\right)^{2}\right), \\
F_{1 j}(\psi)=\omega_{m} d \Delta C_{L_{0 j}} / d \psi, F_{2 j}(\psi)=\left(-0.018 \Delta C_{L_{0 j}}+0.006 \omega_{m} d \Delta C_{L_{0 j}} / d \psi\right) / \tau_{j}^{2},
\end{gathered}
$$

and $\alpha_{z j}$ is the blade section zero-lift angle-of-attack. In matrix form:

$$
\begin{gathered}
\frac{d}{d t}\left[\begin{array}{c}
\Delta \mathbf{C}_{L 1} \\
\Delta \mathbf{C}_{L 2} \\
\Delta \mathbf{C}_{L 2 d}
\end{array}\right]+\left[\begin{array}{ccc}
\mathbf{C}_{10} & \mathbf{0} & \mathbf{0} \\
\mathbf{0} & \mathbf{I} & \mathbf{0} \\
\mathbf{0} & \mathbf{C}_{21} & \mathbf{C}_{20}
\end{array}\right]\left[\begin{array}{c}
\Delta \mathbf{C}_{L 1} \\
\Delta \mathbf{C}_{L 2} \\
\Delta \mathbf{C}_{L 2 d}
\end{array}\right]=\left[\begin{array}{c}
\mathbf{F}_{1}(\psi) \\
\mathbf{0} \\
\mathbf{F}_{2}(\psi)
\end{array}\right], \\
\mathbf{C}_{L}=\left[C_{L j}\right]=\mathbf{C}_{L s}+\Delta \mathbf{C}_{L 1}+\Delta \mathbf{C}_{L 2} .
\end{gathered}
$$

Thus, the propeller load torque is given by Equation (2) and the motor dynamics defined by Equation (24).

\section{Typical Simulation Results}

In this section, typical simulation-based responses of an HTS PMSM-driven propeller are illustrated. The typical parameters of a PMSM-propeller system are listed in Table 1. The parameters corresponding to the case when a motor is within a cryogenic cooler at superconducting temperatures were estimated from the properties of a superconducting HTS tape and flux-trapped magnetic material. The typical properties of a superconducting HTS tape used in HTS PMSM, as well as the field due to flux trapping, were obtained from [10-14]. The stator resistance shown in the table includes the resistance of the source, as well as the stator winding.

Table 1. Assumed parameters of a permanent magnet synchronous motor (PMSM)-propeller system. (The parameters corresponding to a superconducting temperature are indicated by 'sc').

\begin{tabular}{cccc}
\hline Parameter & Value & Parameter & Value \\
\hline Phase voltage (max) & $28 \mathrm{~V}$ & Supply frequency & $60 \mathrm{~Hz}$ \\
$L_{q}$ & $0.0078 \mathrm{H}$ & $L_{d}$ & $0.0078 \mathrm{H}$ \\
Pole pairs & 2 & $R_{S}$ (incl. source resistance) & $2.03 \Omega$ \\
$\psi_{f}$ & $0.616 \mathrm{~Wb}$ & $R_{s}(\mathrm{sc})$ (incl. source resistance) & $0.203 \Omega$ \\
$\psi_{f}(\mathrm{sc})$ & $2 \times 0.616 \mathrm{~Wb}$ & Rotor inertia, $J$ & 0.20095 \\
Rotor damping, $B$ & 0.4675 & Synchronous speed, $\omega_{s}$ & $60 \pi$ \\
Desired electrical speed & $0.7 \times \omega_{s}$ & Number of blade elements & 10 \\
Propeller diameter & $1.1 \mathrm{~m}$ & Taper ratio & 0.8 \\
Root chord & $0.45 \mathrm{~m}$ & AR & 4.07 \\
Hub diameter & $0.125 \times$ propeller diameter & Hub blade twist & $65^{\circ}$ \\
Tip blade twist & $25^{\circ}$ & Blade profile & $\mathrm{NACA} 0024$ \\
$C_{D_{0}}$ & 0.008 & $C_{D_{1}}$ & -0.03 \\
$C_{D_{2}}$ & 0.01 & $C_{D_{m a x}}$ & $1.11+0.018 \mathrm{AR}$ \\
$C_{D s t a l l}$ & 0.1 & $V_{\infty}$ & $30 \mathrm{~m} / \mathrm{s}$ \\
\hline
\end{tabular}

Initially, the PMSM propeller combination was simulated at normal room temperature with no stator $d$ - and $q$-axes voltage inputs. Thus, the $u_{d}$ and $u_{q}$ voltage inputs in the stator $d$ - and $q$-axes were set to zero. Following the open loop simulation, the PMSM was simulated at superconducting temperatures, again with no control inputs. Figure 1 shows the characteristics of a typical loaded synchronous motor. Figure 1 shows the variation of the $q$-axis current $i_{q}$ as a function of time over the timeframe of $1 \mathrm{~s}$ at normal room temperature. Figure 1 also shows the corresponding variation of the $d$-axis current $i_{d}$ as a function of time, to the same scale. Figure 1 also shows the corresponding 
variation of the mechanical rotor speed $\omega_{m}$ as a function of time over the same time frame. Figure 1 finally also shows the corresponding variation of the mechanical angular rotor position $\theta_{m}$ as a function of time.
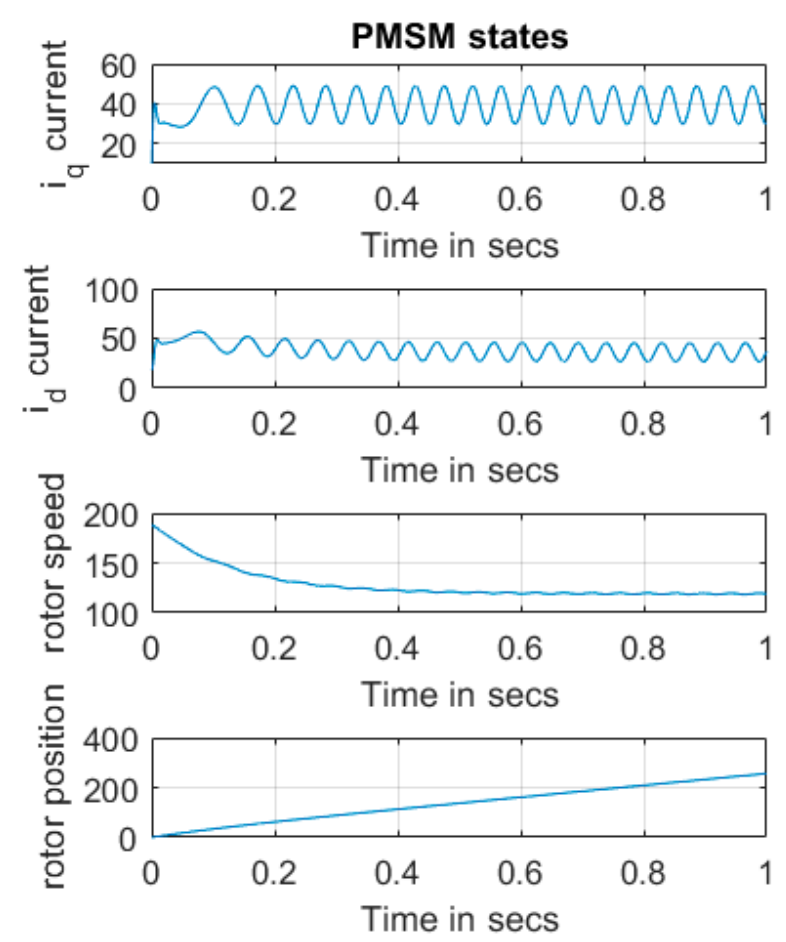

Figure 1. Variation of the permanent magnet synchronous motor (PMSM) characteristics as a function of time over the timeframe of $1 \mathrm{~s}$ at normal room temperature.

Figure 2 shows the variation of the dimensional load torque in Newton-meters as the motor starts and reaches steady state. Figure 3 shows the corresponding non-dimensional characteristics of the propeller, namely the coefficients of thrust and torque, the propulsive efficiency, and the advance ratio as functions of time over the timeframe of $1 \mathrm{~s}$ at normal room temperature. Figures $4-6$ show the same characteristics as in Figures 1-3 but for the HTS motor.

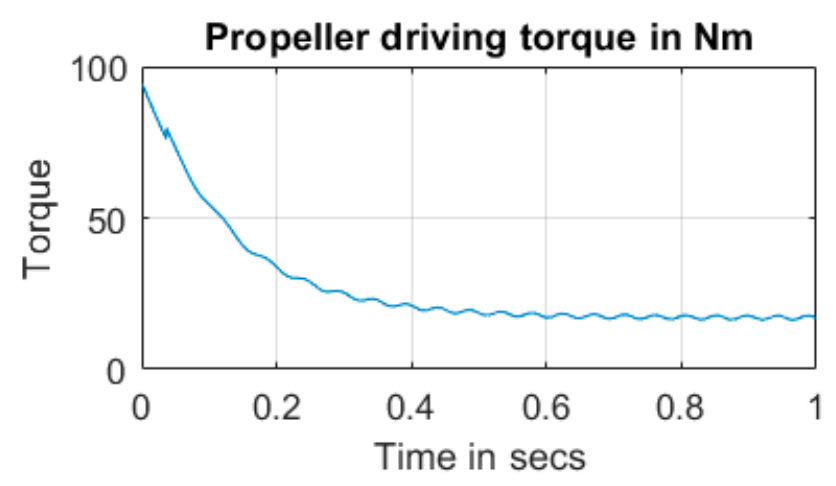

Figure 2. Variation of the PMSM load torque as a function of time over the timeframe of $1 \mathrm{~s}$ at normal room temperature. 

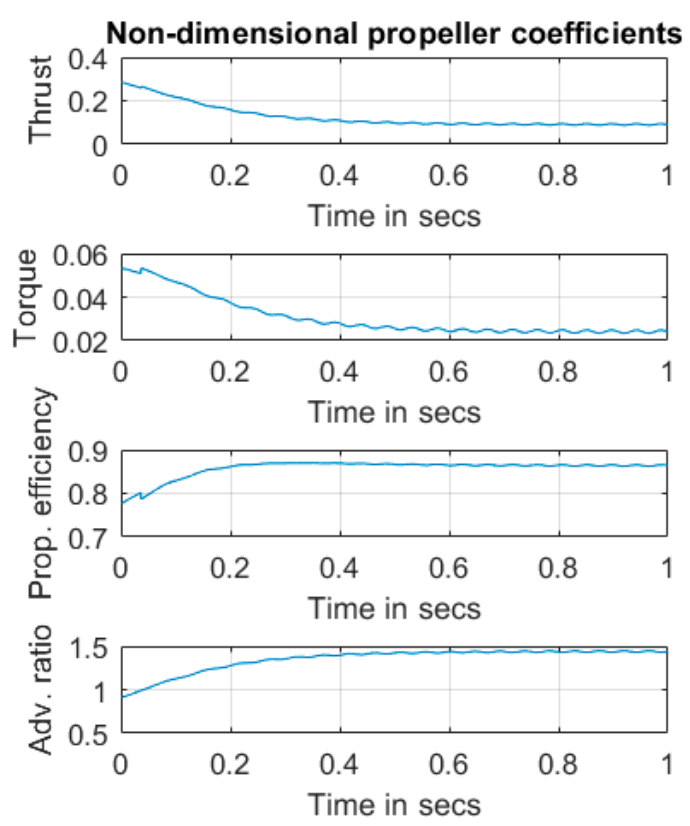

Figure 3. Variation of the PMSM-driven propeller non-dimensional coefficients of thrust and torque, propulsive efficiency, and advance ratio as a function of time over the timeframe of $1 \mathrm{~s}$ at normal room temperature.
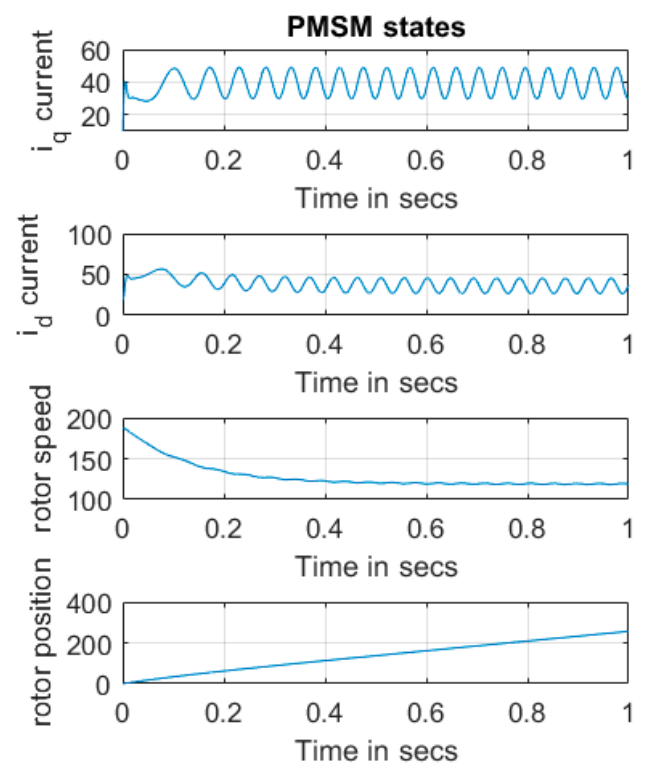

Figure 4. Variation of the high-temperature superconductor (HTS) PMSM characteristics as a function of time over the timeframe of $1 \mathrm{~s}$ at the superconducting temperature. 


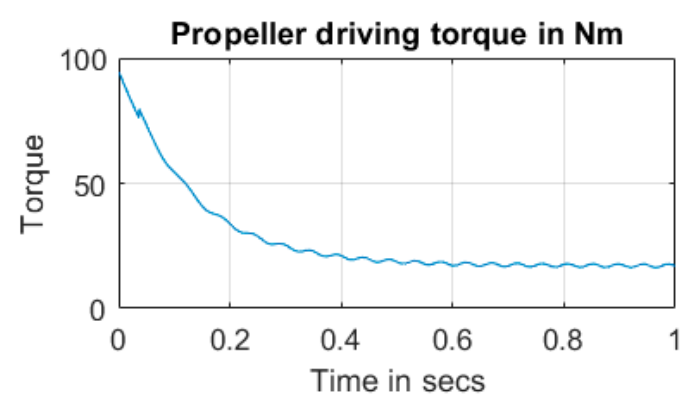

Figure 5. Variation of the HTS PMSM load torque as a function of time over the timeframe of $1 \mathrm{~s}$ at the superconducting temperature.
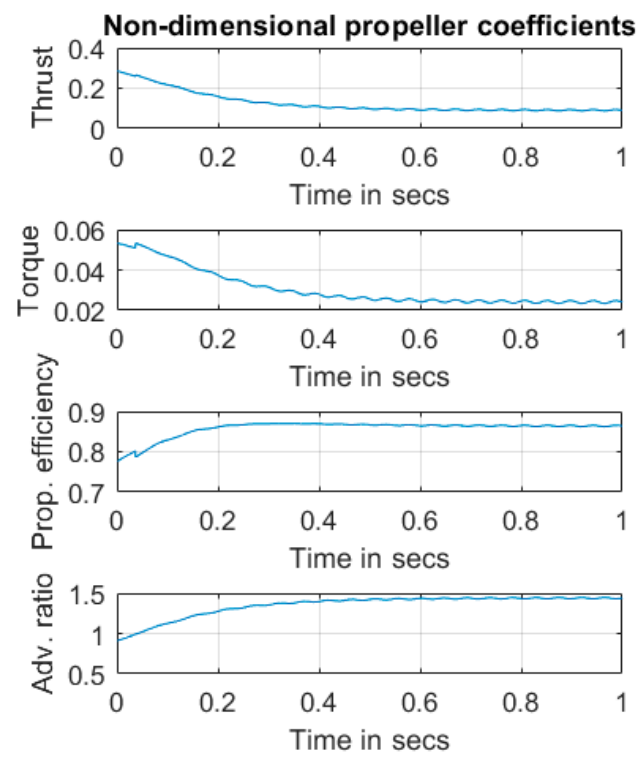

Figure 6. Variation of the HTS PMSM-driven propeller non-dimensional coefficients of thrust and torque, propulsive efficiency, and advance ratio as a function of time over the timeframe of $1 \mathrm{~s}$ at the superconducting temperature.

\section{Discussion and Conclusions}

The above study demonstrates that HTS motors - that is, motors with armature and field windings made of HTS wires or tapes that are encased within a cryostat so that the temperature of the stator and rotor are in the superconductivity domain-may be modeled just as conventional motors. It must be recognized though that the values of the resistances, torque, and back EMF constants are substantially different from the corresponding values in a conventional motor.

The simulation of HTS PMSM was carried out in this paper with the aim of studying the nature of the control strategies that must be applied to a PMSM driving a propeller. The traditional method of controlling a PMSM is to use the strategy of field-oriented control (FOC). FOC is generally implemented by resolving the phase currents in the directions of the direct and quadrature axes, so the current in one axis generates the driving torque while the other generates the flux. One method of implementing such a control law is to use a voltage source inverter. A voltage source inverter (VSI) drive provides a voltage source as compared to a load commutated inverter (LCI) drive, which provides a current source.

The simulations carried out in this paper indicate that it is quite important to drive the propeller at an advance ratio such that it is always operating at the maximum propulsive efficiency point. Moreover, it is also important to operate the propeller while minimizing the overall energy losses. As precision positioning or speed controls are not really required, it was found that the use of a variable frequency drive and a direct current to alternating current power electronic converter may provide the optimum 
energy solution for driving the propeller. However, open loop variable frequency drive-based voltage source control may not be stable for all frequencies and it may be necessary to include a stabilizing inner loop. The stability and inner loop feedback stabilization of a PMSM with an outer open loop variable frequency drive and a constant load have been considered by many authors [25-29] and will not be reconsidered here. Yet, the methodology is particularly suitable for HTS PMSMs, as the stator resistance of these machines is particularly low. Thus, an MPEPT controller, where an outer loop identifies the operating speed at the maximum propulsive efficiency point while the variable frequency drive adjusts the drive frequency so as to force the motor to track the operating speed at the maximum propulsive efficiency point, is essential. For a PMSM, the parameters of the model of the PMSM and the desired advance ratio will influence the selection of the drive frequency. Thus, not only must the parameters of the PMSM be known accurately, it must be ensured that the HTS PMSM is always operating in identical conditions to ensure that the parameters are not changing continuously. Alternatively, one must determine the motor parameters in real-time and adapt the controller accordingly.

Currently, a generic voltage source controller based on a multi-loop control structure and an inner stabilization loop are being developed to control a typical HTS PMSM with a dynamic propeller load that is not constant, and the results of that study will be reported independently. The dynamic model is altered to include the load angle dynamics and the inner loop control law is synthesized by the method reported in [29]. Scaling laws for matching the results of this study to other HTS PMSMs are used to facilitate the design of a distributed propulsion system for an all-electric aircraft.

Conflicts of Interest: The authors declare no conflict of interest.

\section{References}

1. Bednorz, J.G.; Müller, K.A. Possible high $T_{\mathrm{c}}$ superconductivity in the Ba-La-Cu-O system. Z. Phys. B 1986, 64, 189-193. [CrossRef]

2. Wu, M.K.; Ashburn, J.R.; Torng, C.J.; Hor, P.H.; Meng, R.L.; Gao, L.; Huang, Z.J.; Wang, Y.Q.; Chu, C.W. Superconductivity at $93 \mathrm{~K}$ in a New Mixed-Phase Y-Ba-Cu-O Compound System at Ambient Pressure. Phys. Rev. Lett. 1987, 58, 908-910. [CrossRef] [PubMed]

3. Ford, P.J.; Saunders, G.A. The Rise of the Superconductors; CRC Press: Boca Raton, FL, USA, 2005.

4. Goyal, A. Second-Generation HTS Conductors; Kluwer Academic Publishers: Dordrecht, The Netherlands, 2005.

5. Bardeen, J.; Cooper, L.N.; Schrieer, J.R. Microscopic theory of superconductivity. Phys. Rev. 1957, 106, $162-164$. [CrossRef]

6. Bardeen, J.; Cooper, L.N.; Schrieer, J.R. Theory of superconductivity. Phys. Rev. 1957, 108, 1175-1204. [CrossRef]

7. Joshi, C.H.; Prum, C.B.; Schiferl, R.F.; Driscoll, D.I. Demonstration of two synchronous motors using high temperature superconducting field coils. IEEE Trans. Appl. Supercond. 1995, 5, 968-971. [CrossRef]

8. Frank, M.; Frauenhofer, J.; Van Hasselt, P.; Nick, W.; Neumueller, H.W.; Nerowski, G. Long-term operational experience with first Siemens $400 \mathrm{~kW}$ HTS machine in diverse configurations. IEEE Trans. Appl. Supercond. 2003, 13, 2120-2123. [CrossRef]

9. Eckels, P.W.; Snitchler, G. 5 MW high temperature superconductor ship propulsion motor design and test results. Nav. Eng. J. 2005, 117, 31-36. [CrossRef]

10. Masson, P.J.; Soban, D.S.; Upton, E.; Pienkos, J.E.; Luongo, C.A. HTS motors in aircraft propulsion: Design considerations. IEEE Trans. Appl. Supercond. 2005, 15, 2218-2221. [CrossRef]

11. Neumuller, H.W.; Nick, W.; Wacker, B.; Frank, M.; Nerowski, G.; Frauenhofer, J.; Rzadki, W.; Hartig, R. Advances in and prospects for development of high-temperature superconductor rotating machines at Siemens. Supercond. Sci. Technol. 2006, 19, S114-S117. [CrossRef]

12. Oswald, B.; Best, K.-J.; Soell, M.; Duffner, E.; Gawalek, W.; Kovalev, L.K.; Krabbes, G.; Prusseit, W. HTS motor program at OSWALD, present status. IEEE Trans. Appl. Supercond. 2007, 17, 1583-1586. [CrossRef]

13. Kalsi, S.S. Rotating AC machines. In Applications of High Temperature Superconductors to Electric Power Equipment; John Wiley \& Sons, Inc.: Hoboken, NJ, USA, 2011; Chapter 4. 
14. Kwon, Y.K.; Kima, H.M.; Baik, S.K.; Lee, E.Y.; Lee, J.D.; Kim, Y.C.; Lee, S.H.; Hong, J.P.; Jo, Y.S.; Ryu, K.S. Performance test of a $1 \mathrm{MW}$ class HTS synchronous motor for industrial application. Phys. C Supercond. 2008, 468, 2081-2086. [CrossRef]

15. Ishmael, S.; Goodzeit, C.; Masson, P.; Meinke, R.; Sullivan, R. Flux pump excited double-helix rotor for use in synchronous machines. IEEE Trans. Appl. Supercond. 2008, 18, 693-696. [CrossRef]

16. Luongo, C.A.; Masson, P.J.; Nam, T.; Mavris, D.; Kim, H.D.; Brown, G.V.; Waters, M.; Hall, D. Next generation more-electric aircraft: A potential application for HTS superconductors. IEEE Trans. Appl. Supercond. 2009, 19, 1055-1068. [CrossRef]

17. Kovalev, K.L.; Dezhin, D.S.; Kovalev, L.K.; Poltavets, V.N.; Ilyasov, R.I.; Golovanov, D.S.; Oswald, B.; Best, K.-J.; Gawalek, W. HTS high-dynamic electrical motors. Available online: http:/ / snf.ieeecsc.org/sites / ieeecsc.org/files/EUCAS2009-ST152.pdf (accessed on 12 February 2018).

18. Oswald, B.; de Waele, A.T.A.M.; Söll, M.; Reis, T.; Maier, T.; Oswald, J.; Teigelkötter, J.; Kowalski, T. Project Sutor: Superconducting speed-controlled torque motor for $25.000 \mathrm{Nm}$. Phys. Procedia 2012, 36, 765-770. [CrossRef]

19. Armstrong, M.; Ross, C.; Phillips, D.; Blackwelder, M. Stability, Transient Response, Control, and Safety of a High-Power Electric Grid for Turboelectric Propulsion of Aircraft; NASA/CR-2013-217865; NASA: Hanover, MD, USA, 2013.

20. Tsukamoto, O. Present status and future trends of R\&D for HTS rotational machines in Japan. Phys. C Supercond. 2014, 504, 106-110.

21. Okazaki, T.; Sugimoto, H.; Takeda, T. Liquid nitrogen cooled HTS motor for ship propulsion. In Proceedings of the Power Engineering Society General Meeting, Montreal, QC, Canada, 18-22 June 2006.

22. Rambabu, S. Modeling and Control of a Brushless DC Motor. Master's Thesis, Department of Electrical Engineering, National Institute of Technology, Rourkela, India, 2007.

23. Vepa, R. Dynamic modelling simulation and control of energy generation. In Wind Power Generation and Control; Lecture Notes in Energy Series No. 20; Springer Verlag: London, UK, 2013; Chapter 4.

24. Han, W.; Liu, J.; Liu, C.; Chen, L.; Su, X.; Zhao, P. Flap motion of helicopter rotors with novel, dynamic stall model. Open Phys. 2016, 14, 239-246. [CrossRef]

25. Perera, P.D.C.; Blaabjerg, F.; Pedersen, J.K.; Thgersen, P. A sensorless, stable V/f control method for permanent-magnet synchronous motor drives. IEEE Trans. Ind. Apps. 2003, 39, 783-791. [CrossRef]

26. Agarlita, S.C.; Coman, C.E.; Boldea, I. Stable V/f control system with controlled power factor angle for permanent magnet synchronous motor drives. IET Electr. Power Appl. 2006, 2, 278-286. [CrossRef]

27. Brock, S.; Pajchrowski, T. Energy-Optimal V/f Control of Permanent Magnet Synchronous Motors for Fan Applications. Zesz. Probl. Masz. Elektr. 2011, 92, 169-174.

28. Stellas, D. Sensorless Scalar and Vector Control of a Subsea PMSM. Master's Thesis, Department of Energy and Environment, Division of Electric Power Engineering, Chalmers University of Technology, Göteborg, Sweden, 2013.

29. Paitandi, S.; Sengupta, M. Analysis, design, implementation of sensorless V/f control in a surface mounted PMSM without damper winding. Sādhanā 2017, 42, 1317-1333. [CrossRef]

(C) 2018 by the author. Licensee MDPI, Basel, Switzerland. This article is an open access article distributed under the terms and conditions of the Creative Commons Attribution (CC BY) license (http:/ / creativecommons.org/licenses/by/4.0/). 